\title{
65-Year-Old Man with Weight Loss, Anorexia, AND Distal EXTREMity NumbNess
}

\author{
Leigh Van Vranken, MS III, William Kim, MS III, Darren N. Seril, MD, Toshimasa Okabe, MD
}

\section{Case Report}

A 65-year-old Caucasian male with no significant past medical history presented to the emergency department with an unintentional 44-pound weight loss over a four-month period. The weight loss was preceded by fatigue and anorexia, which had been increasing for approximately eight months. In addition, he noted numbness and tingling of his hands and feet that began over the same time period. The patient was initially treated for depression with sertraline by his primary care physician. However, his symptoms persisted without significant improvement. An initial workup at outside hospital one month prior to presentation included an abdominal CT scan, which revealed a mass in the pancreatic head suggestive of a pseudocyst. He was also treated for Lyme disease with a course of doxycycline based on a rash finding on his left thigh. Lyme serologies were negative. He denied fever, chills, night sweats, changes in bowel habits or stool character, dysphagia, odynophagia, abdominal pain, cough, or shortness of breath. $\mathrm{He}$ also denied focal weakness, changes in speech, or changes in vision. He has no known drug allergies. Medications included sertraline for depression, zolpidem and diphenhydramine for sleep. He denied tobacco or illicit drug use. He drinks alcohol occasionally. Family history was noncontributory, with no history of malignancy, neurodegenerative or endocrine disorders.

On examination, patient was afebrile with normal vital signs. The patient appeared cachectic. Body-mass index (BMI) was 18.2 (normal range 18.5 to 24.9 ). Bilateral temporal muscle wasting was noted. Examination of the oral cavity revealed poor dental hygiene, black discoloration of the tongue, and dry mucous membranes. The abdomen was soft, non-tender, non-distended, with normal active bowel sounds. There was no hepatosplenomegaly. Skin was dry and showed decreased turgor. Neurological exam revealed altered sensation in upper and lower distal extremities, most marked on the feet and shins. Muscle strength was normal with the exception of mildly decreased thumb abductor and adductor muscle strength bilaterally. The knee-jerk and Achilles reflexes were present but diminished bilaterally. The remainder of the exam was unremarkable.

\section{Hospital Course}

The initial differential diagnosis for the patient's unintentional weight loss included an occult malignancy, chronic infection or inflammation, malabsorption, and depression. Given the prior finding of a pancreatic cyst, endoscopic ultrasound with fine need aspiration (FNA) was performed, which confirmed the presence of a benign pseudocyst. Esophagogastroduodenoscopy (EGD) and colonoscopy were performed to assess for malabsorption and gastrointestinal cancer. Both studies were unrevealing for diagnosis. Further evaluation for an occult malignancy included CT scans of the mandibular, neck, thorax, and abdomen. There was no evidence of solid tumor or lymphadenopathy from these imaging studies. CEA was $9.6 \mathrm{ng} / \mathrm{mL}$ (normal range: 0.0-5.0 $\mathrm{ng} / \mathrm{mL}$ ) and CA 19-9 was less than $1.0 \mathrm{ng} / \mathrm{mL}$ (normal range: 0.0-36.0 U/mL). Prostate-specific antigen (PSA) was normal. The patient was seen in consultation by psychiatry and it was determined that the patient did not meet criteria for clinical depression or dementia. He was subsequently tapered off of sertraline.

Further evaluation of the complaint of distal extremity numbness and tingling included screens for diabetes, vitamin deficiency, and occult malignancy. Vitamin B12 level was within normal limits. There were no signs of chronic inflammation or multi-organ involvement suggestive of systemic vasculitis. Paraneoplastic syndrome was also considered in the differential. Nerve conduction studies (NCS) and electromyography (EMG) showed impaired nerve conduction, axonal degeneration, and demyelination of both motor and sensory nerves. These findings were suggestive of chronic inflammatory demyelinating polyradiculoneuropathy (CIDP).

The patient's white blood cell (WBC) count was normal to mildly elevated throughout the hospital course. However, lymphocytosis (differential: neutrophil 39.2\%, lymphocyte $53.8 \%$, monocyte $4.3 \%$ ) was noted on admission and persisted. Further evaluation of the peripheral blood smear (PBS) showed large granular lymphocytosis (LGLs) (Figure 1), possibly attributable to reactive phenomenon,but a neoplastic process could not be ruled out. ${ }^{1}$ Flow cytometry showed a large (37\% of all non-erythroid cells) cluster of $\mathrm{CD} 45$ positive cells. ${ }^{2}$ Electronic gating analysis revealed expansion of a natural killer (NK) cell

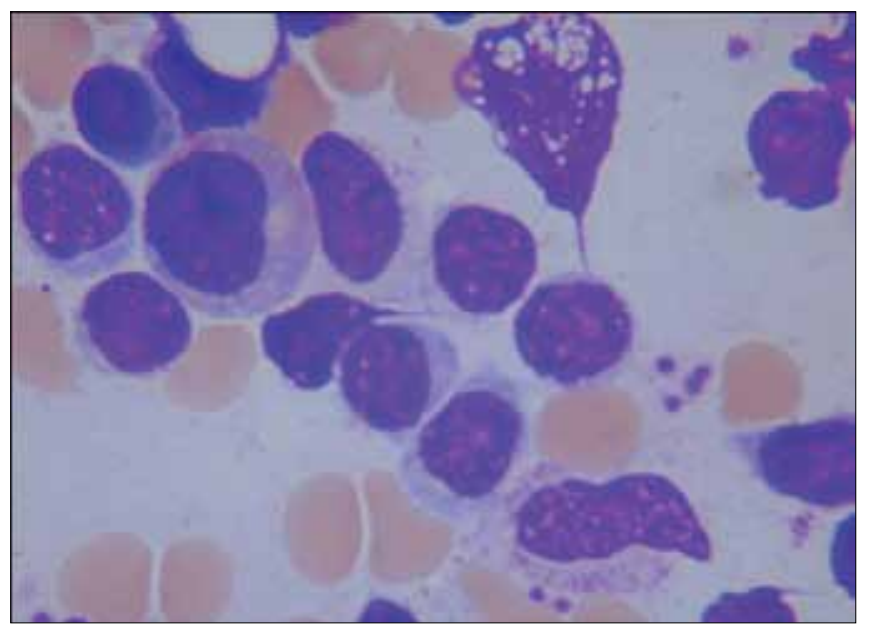

Figure 1. Peripheral blood smear showing large granular lymphocytes (LGL). 
Table 1. Flow Cytometry of peripheral blood lymphocytes.

\begin{tabular}{|l|l|l|}
\hline Marker & Cell type normally expressing thismarker & $\%$ cells expressing marker \\
\hline CD52 & Mature lymphocyte & $99 \%$ \\
\hline CD2 & T cell or NK cell & $97 \%$ \\
\hline CD19 & B cell & $2 \%$ \\
\hline CD20 & B cell & $3 \%$ \\
\hline CD3 & T cells & $12 \%$ \\
\hline CD4 & Helper \& regulatory T cell, macrophage \& monocyte & $9 \%$ \\
\hline CD8 & Cytotoxic T cell \& 30-50\% of NK cell & $3 \%$ \\
\hline CD56 & NK cell & $79 \%$ \\
\hline Kappa light chain & B cell & $77 \%$ \\
\hline
\end{tabular}

population lacking CD8 expression and expressing Kappa light chain, or a molecule immunoreactive with anti-Kappa light chain antibody (Table 1). Kappa light chain is typically expressed by B lymphocytes. The expression of this molecule in a NK cell population was suggestive of clonal expansion.

To investigate the possibility of leukemia, a bone-marrow biopsy was performed, revealing normal marrow architecture with LGLs similar to those observed on PBS. Polymerase chain reaction (PCR) analysis of the T-cell receptor (TCR) gene in peripheral blood samples showed a high frequency single mutation in the TCR-gamma gene, suggestive of clonality.

The patient continued to have a lack of appetite during his hospital stay, as well as inadequate oral intake to meet his nutritional needs. He was administered peripheral parenteral nutrition (PPN) throughout his stay, until a percutaneous endoscopic gastrostomy (PEG) tube was placed. His hospital course was otherwise uneventful and he was discharged to home with PEG tube feeds to supplement his oral nutrition, and arrangements to follow-up with hematology for further management of a hematologic malignancy.

Based on the results of his biopsy, the final diagnosis was natural killer cell large granular lymphocyte leukemia with possible paraneoplastic peripheral neuropathy.

\section{Discussion}

Differential diagnosis for unintentional weight loss with lack of appetite is broad. In the elderly, common causes of weight loss include malignancy, depression, and gastrointestinal diseases. Chronic pulmonary disease and congestive heart failure can cause involuntary weight loss by producing anorexia and increasing resting energy expenditure. In addition, medications such as antibiotics, non-steroidal anti-inflammatory drugs, and serotonin reuptake inhibitors, as well as socioeconomic hardships, can cause profound weight loss. The extensive workup in the present case suggested hematological malignancy as the cause of weight loss.
The causes of peripheral neuropathy are also numerous. Common causes include systemic diseases (i. e. diabetes mellitus, vasculitis), chronic liver disease, nutritional deficiency, infections, neoplasms, medications, and toxins. In our case, the results of NCS and EMG studies were suggestive of CIDP. CIDP is a chronically progressive or relapsing sensorimotor disorder with infiltration by lymphocytes and macrophages into peripheral nerve tissue. ${ }^{3}$ This is the chronic equivalent of acute inflammatory demyelinating polyradiculoneuropathy (AIDP) found in the Guillain-Barré syndrome (GBS). However, unlike GBS, CIDP is usually idiopathic and lacks a strong association with an antecedent bacterial or viral infection. ${ }^{4}$ CIDP may also be part of a paraneoplastic syndrome associated with solid or hematologic malignancy. ${ }^{3}$ In the present case, negative Anti MAG, normal TSH, and cortisol levels and normal protein electrophoresis excluded many of the alternative diagnoses. The occurrence of a peripheral neuropathy in the setting of a hematologic malignancy offers a plausible etiology for the patient's neurologic symptoms (i.e., a paraneoplastic syndrome).

NK-cell leukemia is a disease within a spectrum of disorders ranging from the indolent "chronic NK-cell lymphocytosis" to the quickly fatal "aggressive NK-cell leukemia (ANKL)". The diagnosis of NK-cell leukemia can be difficult to establish because of the lack of readily available clonal markers. Although a subset of NK cells have been shown to express a rearrangement of TCR-gamma, this marker is not reliably present. ${ }^{6}$ The natural history of NK-cell development is incompletely understood. It is currently believed to originate from a common lymphoid progenitor in the bone marrow, but it is also reported that immature thymocytes retain NK-cell potential with an unknown contribution to the steady state pool of NK-cells. ${ }^{7}$ In our case, the expansion of a NK-cell population with a lack of normal CD8 expression raised the question of clonality. The NK-cell population also expressed a unique surface marker for non-B lymphocytes. The marker was either the Kappa light chain or a molecule containing an epitope that cross-reacts with polyclonal antibodies to Kappa light chain. This finding was helpful in 
establishing clonality of the NK-cell population, given the lack of reliable NK-cell-specific markers as noted above.

The clinical presentation of NK-cell leukemia typically includes " $B$ " symptoms (fever, night sweats, weight loss). In addition, other common findings included anemia, thrombocytopenia, hepatosplenomegaly, and gastrointestinal involvement. ${ }^{8}$ The complaints of appetite loss and unintentional weight loss described here represent an indolent and atypical presentation of NK-cell leukemia. To date there is no effective treatment for NK-cell leukemia. Although there is a case series reporting successful allogeneic hematopoietic cell transplantation ${ }^{9}$, the role of this modality in the treatment of NK-cell leukemia is yet to be determined. Most patients with this disorder have a severe and refractory clinical course.

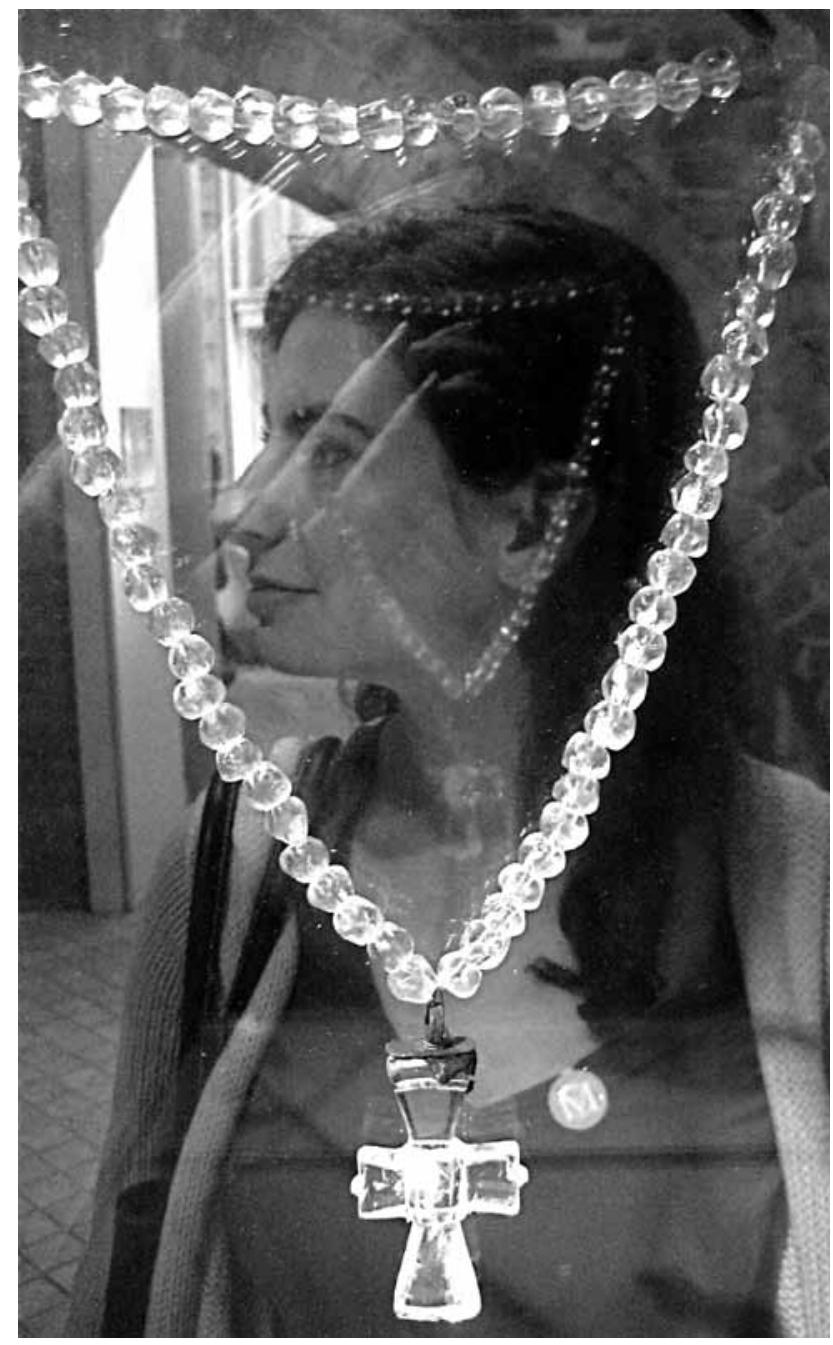

"Metropolitan Museum of Art"

Photograph by Cecilia Kelly, MD

\section{References}

1. Arancha AC, Garcia-Montero J, Almeida Parra P, et al. Potential Role of CMV in the Ontogeny of Monoclonal TCRab+/CD4+ T-Large Granular Lymphocyte [LGL] Lymphocytosis. Haematologica 2007;92[suppl].

2. Morice WG, Kurtin PJ, Leibson PJ, et al. Demonstration of aberrant T-cell and natural killer-cell antigen expression in all cases of granular lymphocytic leukaemia. Br J Haematol 2003;120:1026-36.

3. Yahya C, Nilda T, Burhan T, et al. Chronic Idiopathic Demyelinating Polyneuropathy (CIDP) Associated with Kaposi's Sarcoma. J Neurooncol 2006; 79:323-4.

4. Yuki N, Kuwabara S. Axonal Guillain-Barre syndrome: carbohydrate mimicry and patho-physiology. Journal of the Peripheral Nervous System. 2007;12:238-49.

5. Saperstein, DS. Chronic Acquired Demyelinating Polyneuropathies. Seminars in Neurology. Neuromuscular Disorders. 2008; 28:168-84.

6. Veinotte LL, Greenwood CP, Mohammadi N, et al. Expression of rearranged TCR $\gamma$ genes in natural killer cells suggests a minor thymus-dependent pathway of lineage commitment. Blood 2006;107:2673-9.

7. Oldham R. Natural killer cells: Artifact to reality: An odyssey in biology, Cancer Metastasis Reviews 1983;2:323-36.

8. Sokol L, Loughran TP Jr. Large granular lymphocyte leukemia. Oncologist 2006;11:263-73.

9. Sato E, Ohga S, Kuroda $\mathrm{H}$, et al. Allogeneic hematopoietic stem cell transplantation for Epstein-Barr virus-associated T/natural killer-cell lymphoproliferative disease in Japan. Am J Hematol 2008;83:721-7. 